\title{
NEUROIMAGING IN NEONATAL ENCEPHALOPATHY
}

Investigators at St Francis Medical Center, Cape Girardeau, MO, and multiple centers in the US, using a database from the Vermont Oxford Network Registry, studied the pattern of use and findings of computed tomography, MRI, and intracranial ultrasound in the evaluation of infants with neonatal encephalopathy. CT scans were performed on 933 of 4107 (22.7\%) infants, and 100 of 921 (10.9\%) of those received multiple CT scans. Compared with MRI, CT provided less detailed evaluation of cerebral injury, but was more sensitive than cranial ultrasound for hemorrhage and deep brain structural abnormalities. Despite concerns over potential harm from radiation exposure and a diagnostic value inferior to that of MRI, CT is commonly used for neonatal imaging. Data collected in this study suggest that cranial ultrasound for screening, followed by MRI is more appropriate than CT at any stage of evaluation of infants with neonatal encephalopathy. (Barnette AR, Horbar JD, Soll RF, et al. Neuroimaging in the evaluation of neonatal encephalopathy. Pediatrics 2014 May 26;133(6):e1508-17).

COMMENTARY. Fetal exposure to radiation is linked to neurologic abnormalities [1], and infant medical radiation exposure is associated with impaired cognitive development [2]. The Image Gently education campaign in radiology promotes reduction of the frequency of CT scanning [3], but further efforts by caregivers of newborns are needed to limit the use of CT in evaluation of neonatal encephalopathy [4].

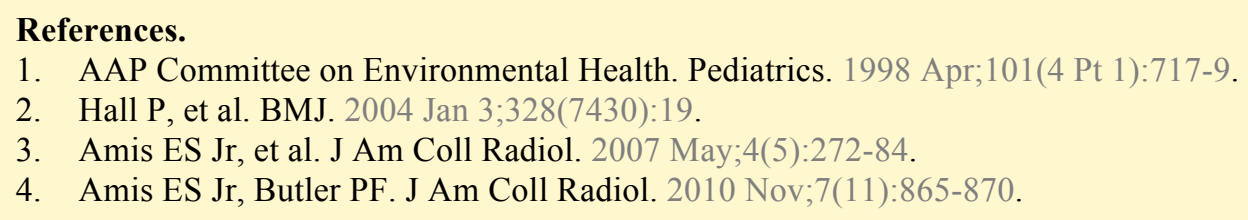

\section{AUTOIMMUNE DISORDERS}

\section{CELIAC DISEASE AND OCCIPITAL EPILEPSY}

Investigators at University of Gaziantep, Turkey, studied the frequency of celiac disease (CD) among children with occipital epilepsy. Two out of 90 epilepsy patients with occipital discharges were tissue transglutaminase (tTG) positive, a prevalence of $1 / 45(2.22 \%)$, and diagnosis of $\mathrm{CD}$ was confirmed by endoscopic duodenal biopsy. In patients with occipital lobe epilepsy associated with $\mathrm{CD}$, seizures were uncontrolled with monotherapy (oxcarbamazepine or valproic acid). Screening for $\mathrm{CD}$ is recommended in children with resistant occipital epilepsy. (Dai AI, Akcali A, Varan C, Demiryurek AT. Prevalence of resistant occipital lobe epilepsy associated with celiac disease in children. Childs Nerv Syst 2014 Jun;30(6):1091-8).

COMMENTARY. Neurological diseases associated with $\mathrm{CD}$ in addition to occipital epilepsy include cerebellar ataxia, peripheral neuropathy, myositis, neuromyotonia, myasthenic syndrome, cerebral calcification, myelopathy, and dementia. Reports of the prevalence of CD in children with occipital epilepsy are variable and 
usually 2 or 3 times higher than the general population. The prevalence of $1 / 45$ quoted by the present Turkish group was not statistically significant [1]. In one large-scale study, the frequency of CD confirmed by the antiendomysial antibody IgA scan and small intestine biopsy was $1 / 127$ in epileptic patients $(n=255)$ and $1 / 293$ in a control group $(n=3,400)[2]$.

\section{References.}

1. Dai AI, et al. Childs Nerv Syst. 2014 Jun;30(6):1091-8.

2. Pratesi R, et al. Arq Neuropsiquiatr. 2003 Jun;61(2B):330-4.

\section{EPILEPSY AS AN AUTOIMMUNE DISEASE}

Investigators at University of New South Wales, Sydney, Australia, and Boston Children's Hospital, Harvard Medical School, conducted a retrospective population-level study of the relationship between epilepsy and 12 common autoimmune diseases: type 1 diabetes mellitus, psoriasis, rheumatoid arthritis, Graves disease, Hashimoto thyroiditis, Crohn disease, ulcerative colitis, systemic lupus erythematosus, antiphospholipid syndrome, Sjogren syndrome, myasthenia gravis, and celiac disease. The risk of epilepsy was significantly heightened among patients with all autoimmune diseases $(\mathrm{P}<0.001)$, and especially in children. Children with autoimmune diseases had a 5-fold increased risk of epilepsy. (Ong M-S, Kohane IS, Cai T, Gorman MP, Mandl KD. Population-level evidence for an autoimmune etiology of epilepsy. JAMA Neurol 2014 May;71(5):56974).

COMMENTARY. The authors theorize that the occurrence of epilepsy in patients with autoimmune disease (AD) might be attributable to the inflammatory component of AD [1]. Also, certain anticonvulsant drugs, such as valproate and carbamazepine, are anti-inflammatory, and some anti-inflammatory drugs have anticonvulsant properties [2].

Investigators at the Lurie Children's Hospital of Chicago have provided several reports of the role of brain inflammation in epileptogenesis. Cortical tissue collected from children with intractable epilepsy at time of surgery showed active neuroinflammation and marked cellular injury [3]. In a review of brain inflammation in the pathogenesis of epilepsy, common pediatric autoimmune diseases are implicated, and the effective use of anti-inflammatory treatments of intractable epilepsy, such as steroids, is documented [4].

\section{References.}

1. Ong M-S, et al. JAMA Neurol. 2014 May;71(5):569-74.

2. Marchi N, et al. PLoS One. 2011 Mar 28;6(3):e18200.

3. Choi J, Nordli DR Jr, Alden TD, et al. J Neuroinflammation. 2009 Dec 19;6:38.

4. Choi J, Koh S. Yonsei Med J. 2008 Feb 29;49(1):1-18.

\section{MAINTENANCE THERAPY FOR MYASTHENIA GRAVIS}

Investigators at Boston Children's Hospital and at University of Florida, Gainesville, studied the comparative efficacy of plasmapheresis (PLEX) vs immunoglobulin (IVIG) as maintenance therapy in juvenile myasthenia gravis (MG). A retrospective analysis over a 33-year period involved 54 children and adolescents with 